\title{
Dissection of a Singularity: The Impact of COVID-19 on Aviation
}

Author(s): Xiaoqian Sun, Sebastian Wandelt, Anming Zhang

Source: Journal of International Logistics and Trade 2020; 18(3):113-125

Published by: Jungseok Research Institute of International Logistics and Trade, Inha University

DOI: https://doi.org/10.24006/jilt.2020.18.3.113

The Journal of International Logistics and Trade is an official journal published by Jungseok Research Institute of International Logistics and Trade, Inha University, Korea. JILT welcomes manuscripts that advance the practice and science of logistics, trade, and other related fields.

Frequency: Quarterly (March, June, September, December)

Stable URL: https://www.ejilt.org

The Jungseok Research Institute of International Logistics and Trade is a specialized academic research institute representing Inha University and the Inha Foundation in Korea. The institute aims to become a representative institute in Northeast Asia in the research of logistics and trade.

Stable URL: https://jrieng.inha.ac.kr

(C) Copyright. Jungseok Research Institute of International Logistics and Trade.

This is an Open-Access article distributed under the terms of the Creative Commons Attribution NonCommercial License (http://creativecommons.org/licenses/by-nc/4.0/) which permits unrestricted noncommercial use, distribution, and reproduction in any medium, provided the original work is properly cited 


\title{
Journal of International Logistics and Trade
}

J. Int. Logist. Trade, 2020, Vol. 18, No. 3, 113-125

https://doi.org/10.24006/jilt.2020.18.3.113

pISSN : 1738-2122 eISSN : 2508-7592

https://www.ejilt.org

\section{ARTICLE}

\section{Dissection of a Singularity: The Impact of COVID-19 on Aviation}

\author{
Xiaoqian Sun ${ }^{1}$, Sebastian Wandelt ${ }^{1,}{ }^{*}$, Anming Zhang ${ }^{2}$ \\ ${ }^{1}$ School of Electronic and Information Engineering, Beihang University, Beijing, China \\ ${ }^{2}$ Sauder School of Business, University of British Columbia, Vancouver, BC, Canada
}

\author{
Received August 01, 2020 \\ Revised September 06, 2020 \\ Accepted September 14, 2020 \\ *Corresponding author: Sebastian Wandelt \\ School of Electronic and Information \\ Engineering, Beihang University, Beijing, \\ China \\ Tel: +86-1012356347 \\ E-mail: wandelt@informatik.hu-berlin.de
}

\begin{abstract}
The current outbreak of COVID-19 is an unprecedented event in air transportation. In this study, we investigate the impact of COVID-19 on global air transportation through the lens of complex networks different at different scales, ranging from worldwide airport networks where airports are nodes and links between airports exist when direct flights exist, to international country networks where countries are contracted as nodes, and to domestic airport networks for representative countries/regions. We focus on the spatial-temporal evolutionary dynamics of COVID-19 in air transportation networks, discovering hidden patterns on flight frequency reduction. Our study provides a comprehensive empirical analysis on the impact of the COVID-19 pandemic on aviation from a complex system perspective.
\end{abstract}

Keywords Air transportation, COVID-19, Complex networks

\section{Introduction}

In recent years, the aviation industry has been jumping from one peak to another, with historical and future predicted annual growth rates between 2\% and 5\% (Janic 2000; Lee et al. 2009). Yet, in the end of 2019, nobody would have expected that large parts of the global aviation system would be taken down within months, in a historically unprecedented manner. The enhanced mobility of aviation, owing to increased connectivity and efficiency (derived partly from agglomeration and traffic density effects), has become a two-edged sword. While air mobility has allowed more passengers to fly to more and remote destinations within a few hours at very low prices (Diaconu 2012), it has also significantly contributed to the risk of spreading diseases worldwide. It has been well known that air transportation plays a critical role in the spread of contagious diseases worldwide (Brockmann and Helbing 2013); typical examples include the outbreaks of SARS 2003 (Likhacheva 2006), MERS 2012 (Zaki et al. 2012), as well as Ebola 2014 (Bogoch et al. 2015). While all these previous diseases have had terrible and harmful effects on their own, these consequences occurred more directly at the local level. The international community and organizations were able to cut the transmission chain early, which ended the localized epidemics before they could turn into a full pandemic. Despite of heavy travel ban restrictions as well as quarantine policies enforced by governments, the COVID-19 outbreak, which is believed to have started around January 2020, quickly spread to almost all countries worldwide. The number of infected cases reached one million in April and surpassed 5.8 million in late May, with the disease killing more than 360,000 people worldwide. One could say that this pandemic has affected the life of almost every person on this planet. Strict travel ban restrictions further amplified the substantial reduction of air passenger demand and resulted in a large number of flight cancellations. Although the total number of the COVID-19 infected cases is surging, a few countries seem to have achieved certain successes in fighting COVID-19, such as China where rebound activities have been undertaken in order to recover the economic development.

The ambivalence of aviation, as the major driver of the COVID-19 outbreak internationally and one of the major (economic) casualties, turns this event into a singularity of historical dimension for aviation. Overall, ICAO estimated that the aviation industry would lose approximately up to USD 418 billion, and the air passenger traffic encountered an unprecedented decline ever since the WWII in 1945. As of May 2020, most countries have active flight bans. More than half of all countries worldwide still have full or partial flight restrictions and that the mobility of air passengers is heavily constrained, particularly for international air travelers. For instance, initiated at the end of March 2020, China is continuing its Five Ones flight restriction policy, meaning that each country can only have one airline to and from China via one route, and one flight per week. When 
the Five Ones Policy will be lifted remains unclear.

In this study, we investigate the impact of the COVID-19 pandemic outbreak on global air transportation. For this purpose, we analyze the aviation system in the period from January 2020 to May 2020. Our analysis is mostly based on operational indicators and complex network indicators. Traditionally, the global aviation system is analyzed as an airport network, with nodes being airports and flights representing the direct connections between airports. While we do investigate this traditional view, we also look at two particularities of air transportation in detail. From the observed reactions of countries regarding flight bans, we can see that there is no clear strategy worldwide; for some countries, one could say that they make arbitrary, politicallyinfluenced decisions on which passengers/aircraft are allowed to enter a country. Given the instrumental role of countries as decision makers, we also investigate the so-called country network, where nodes are countries and links denote the existence of direct flights between these countries. This aggregated network sheds light on the evolution of international connectivity during the COVID-19 outbreak so far. A complementary view of the country network is the domestic airport networks of countries. By looking at the difference between international flight bans and domestic flight reductions, we can obtain insights regarding the overall coherence of countries' decisions. Overall, the focus of our study is on the spatial-temporal evolutionary dynamics of the COVID-19 pandemic on air transportation networks. We do not aim to provide an in-depth analysis of global aviation as a driver in the spread of COVID-19; the reader is referred to other recent studies on this topic (Christidis and Christodoulou 2020; Gilbert and et al. 2020; Zhang et al. 2020). Our work contributes towards providing a complete picture of the COVID-19 outbreak and its effect on aviation.

The remainder of this study is organized as follows. Section 2 discusses the relevant literature on air transportation networks, with a specific focus on disease spreading. Section 3 reports the results of the impact of the COVID-19 pandemic on air transportation. Finally, we summarize the major findings of the paper and discuss future research directions in Section 4.

\section{Literature review}

Complex networks are often used to uncover hidden properties in real-world systems. Particularly, network science tools have been shown to be extremely useful to better understand the structures and dynamics of disease spreading on complex networks. Brockmann and Helbing (2013) proposed a novel notion of distance, the so-called effective distance, derived from the underlying mobility network, instead of the conventional geographic distance; with the goal to investigate the hidden geometry of complex, network driven contagion phenomena. This approach was applied to the worldwide 2009 H1N1 influenza pandemic and 2003 SARS epidemic. Iannelli et al. (2017) proposed a random walk effective distance to predict disease arrival times on complex networks instead of the most probable path. Results showed that the generalized effective distance overcame the restriction of simple path propagation of a disease and the shortest-path measure is a particular case of the proposed random walk effective distance. Meanwhile, Colizza et al. (2006) presented a stochastic computational framework for the forecast of global epidemics based on the IATA database for the year 2002, focusing on the interplay between the network topological structure and the stochastic features of the infection dynamics. With the advent of COVID-19, several studies focused on epidemiological modelling of infectious diseases spreading. Christidis and Christodoulou (2020) presented a methodology during the early stages of the COVID-19 pandemic to measure the risk of the disease spreading outside China, based on the Sabre database tracking the number of passengers from the year 2016 to 2019. The number of reported cases was compared to the estimated traffic density and an indicator of the expected rate of infection of aviation passengers was derived. While focusing on the initial phases of the pandemic, the risks associated with local infections were underestimated, and the asymptomatic carriers could not be measured given that COVID-19 has a long incubation period (up to or even exceeding 14 days). Gilbert and et al. (2020) evaluated the preparedness and vulnerability of African countries against the risk of importation of the COVID19. They found that some African countries with the highest importation risk have moderate to high capacity to respond to the COVID-19 outbreak, while those at moderate risk have variable capacity and high vulnerability. Other studies put an emphasis on the role of multi-modal transportation impacts related to COVID-19, with a focus on China. Zhang et al. (2020) examined the factors influencing the number of imported cases, together with the speed and the spread pattern, of the COVID-19 pandemic spread in China, taking into account different modes of transportation (high-speed rails, coach, and air services). They found that the frequencies of high-speed rails and air services out of Wuhan are significantly associated with the number of the COVID-19 reported cases in the destination cities; the presence of high-speed rail stations and airports is also significantly related to the spread speed of the pandemic.

Another relevant direction in the literature concerns the use of complex network techniques for analyzing the robustness of air transportation systems. While Sun et al. (2017a) analyzed the robustness of air transportation system by understanding the 
node-level metrics, Sun et al. (2017b) investigated the role of network robustness for air transportation systems from the perspective of fractality. See (Wandelt et al. 2018) for an extensive review on dismantling of complex networks.

In summary, the existing literature either a) reports on the air transportation systems before COVID-19 or b) the role aviation plays on the spread of diseases in general; or specifically related to COVID-19. Our study, on the other hand, provides a comprehensive empirical analysis on the impact of the COVID-19 pandemic on aviation from a complex system perspective using network science tools.

\section{Results}

Section 3.1 summarizes the steps for preparing the data and experiments in this study. Section 3.2 discusses the impact of COVID-19 on the worldwide airport network. Section 3.3 is devoted to a different level of abstraction, where nodes in the network are countries and links presents international flights. Finally, Section 3.4 reports on the impact of COVID-19 in individual domestic networks.

\subsection{Data preparation}

The data for this study was obtained from Flightradar24 and prepared by airline. Flightradar24 provides the historical trajectories of registered aircraft and the airline they belong to. All data was downloaded and cleansed according to standard procedures. In total, our dataset covers services of 150 airlines between 2,751 airports. All flights were grouped by days into 24-hour intervals; yielding one network per day induced by the flights taking place on that day. In total, we retrieved data for 152 days, from December 16th, 2019 to May 15th, 2020.

\subsection{Worldwide airport network}

Figure 1 visualizes the worldwide airport networks (Wandelt et al. 2019) on four selected dates before and throughout COVID-19. These snapshots show the airport-level connectivity, with links representing direct flights between airports. We can observe that the Southern hemisphere is more affected than the Northern part. In the middle of May, the Southern region is almost flight-free, compared to earlier days. The worldwide airport network seems largely unchanged for the first 2-3 months of the pandemic. This is rather striking, given that it is well-known that air transportation is the major catalyst for the wider
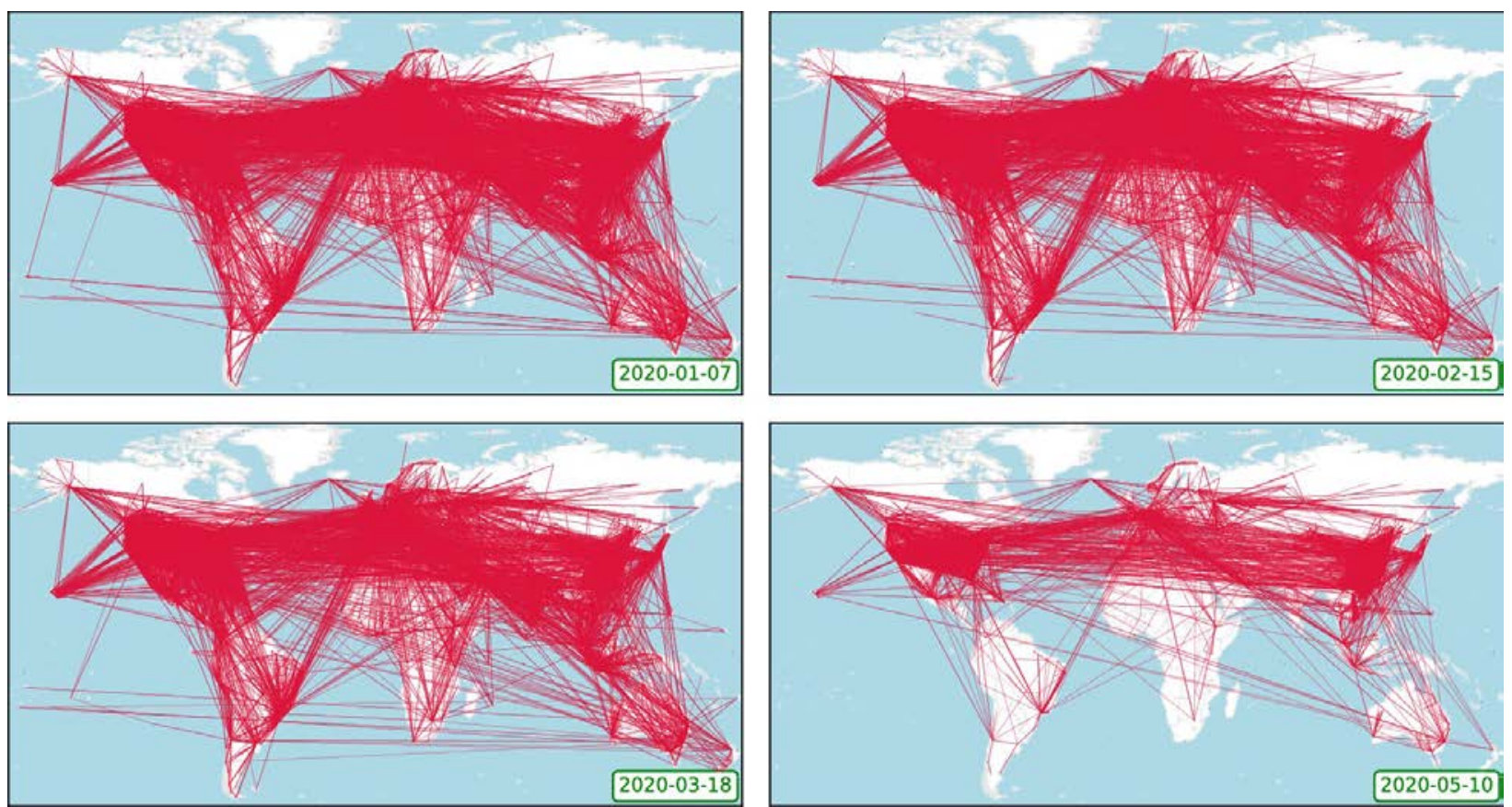

Figure 1. Evolution of the global airport network on four select dates before and during the COVID-19 pandemic. Nodes represent airports and links represent direct flight connections; the width of a link corresponds to the number of direct flights between the airports. 
spread of diseases; one would expect that drastic measures to reduce the connectivity on global transportation would had taken place much earlier.

In order to further investigate the time of significant changes in the worldwide airport network, Figure 2 reports the number of OD (origin-destination) pairs in the network as well as the number of active aircraft over time. Here, an aircraft is labeled as active if it had at least one recorded flight per day. Despite certain seasonal and weekly variations, the airport network was rather stable until the middle of March. The slight trend of reduction in February is mainly caused by the lock-down in China and parts of Asia; however, most other countries had not yet introduced any travel restrictions. Starting from the middle of March, the number of served OD pairs dropped to about one-third of the original number (from 80,000 to around 20,000) within a period of about two weeks. Similarly, the number of active aircraft dropped with a delay of less than a week to a value of around one-fourth of the original traffic (from more than 18,000 aircraft to about 6,000 aircraft). Since the first half of April, the number of OD pairs and flights has been rather stable again, with some typical weekly variation. These analyses show that the aviation world has (measurably) reacted with a delay of about two months to the pandemic; a postponement, which possibly gave away the opportunity to avoid COVID-19 turning into a pandemic with such an extraordinary extent.

The number of active aircraft as well as the number of OD pairs have changed significantly during the pandemic; yet, it is important to understand which of the OD pairs were most affected by the change. Figure 3 reports the distance between served OD pairs throughout the period. It can be seen that the distances between OD pairs were reduced slightly with the introduction

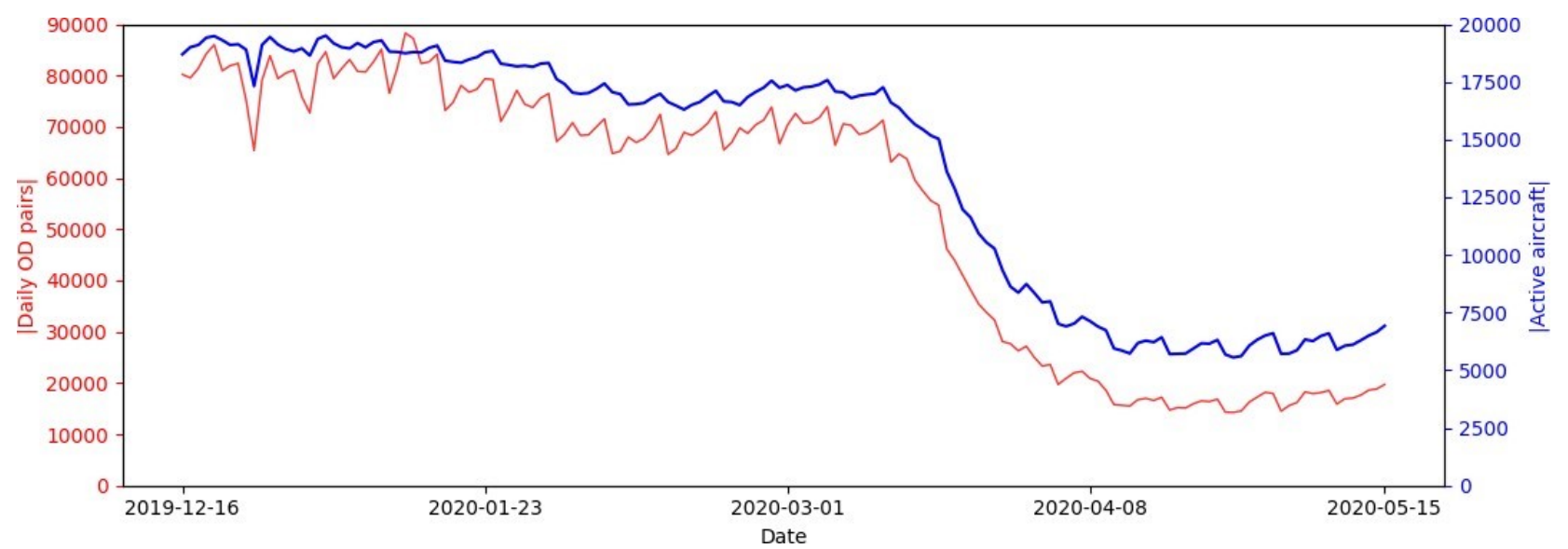

Figure 2. Trend of the number of daily OD pairs (in red color) and the number of active aircraft (in blue color) per day. OD, origindestination.

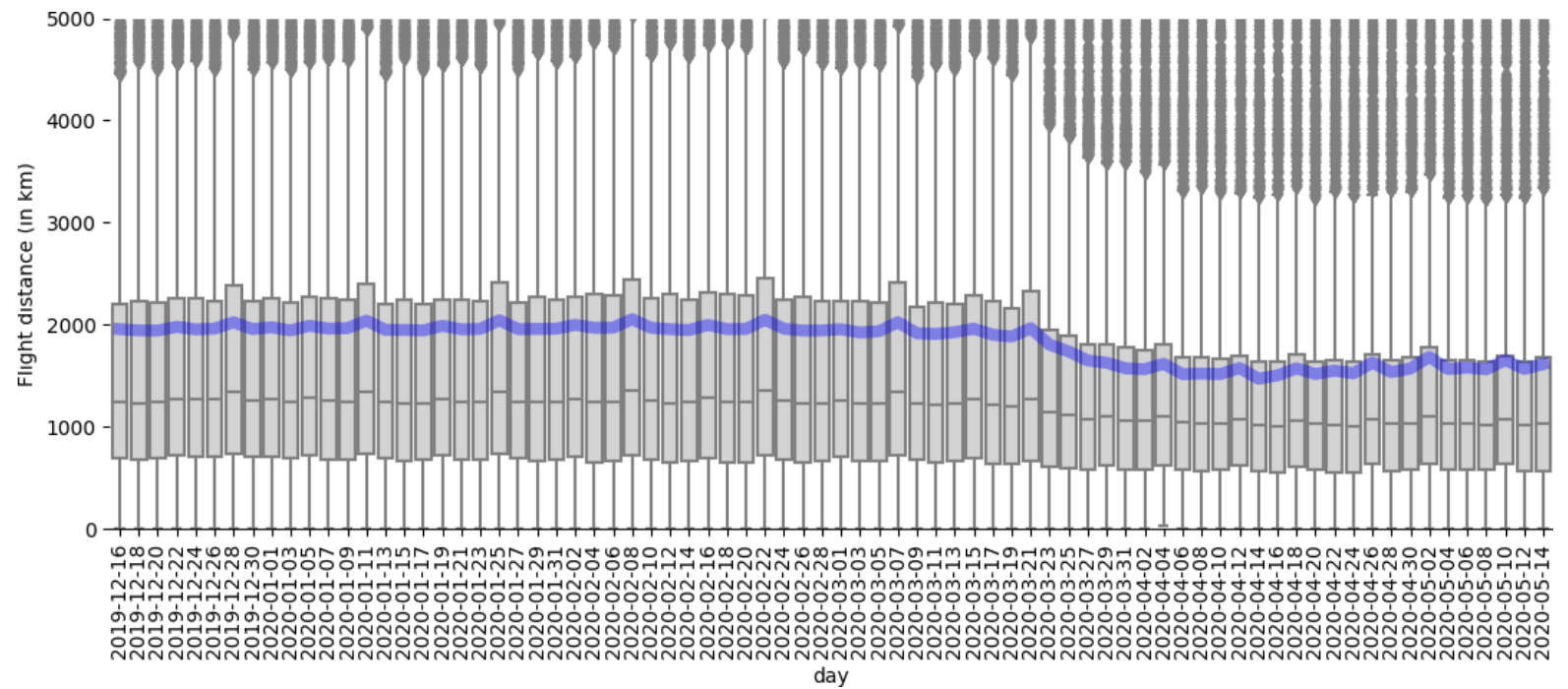

Figure 3. Visualization of the distances (between served airports) flown before and during the pandemic. The boxes represent daily distributions of distances (horizontal black line is the daily median). The shaded blue line is the average distance per day. 
of major flight restrictions, suggesting that mainly long-distance connections have been canceled. However, the effect is much smaller than excepted, given the large drop of OD pairs (to about one- fourth). Accordingly, there should exist additional drivers for the removal process of OD pairs. We will analyze these drivers in later parts of this study. Another question concerns the usage efficiency of aircraft throughout the pandemic. Figure 4 visualizes the number of flights per day per aircraft as a box plot. It can be seen that before the pandemic, the median number of flights per aircraft is four, with a box interval between three and six. Towards May 2020, the median number of flights is reduced to three, with a box interval of two to four. Note that these statistics only cover the active aircraft; non-active aircraft are not included as part of the distribution.

Accordingly, one can conclude that the aircraft efficiency has fallen over time. One reason could be longer turn-around times at airports. However, given the reduced frequencies of flights and since we know that the OD distances did not increase, but rather shrank, it is noteworthy to realize that the chaos of the pandemic significantly reduces the number of flights per aircraft. Finally, it should be noted that the average and median number of flights are rather close to one another throughout the whole period.

In our next analysis, the worldwide airport network is investigated from a complex system perspective (Sun et al. 2017a). We have computed the measure of six frequently-used network properties; Table 1 gives an overview on these network metrics. Figure 5 shows the temporal evolution of these six properties before and throughout the pandemic. The degree centrality of a node measures the fraction of other airports to which a node is connected. The degree centrality drops by around $50 \%$ throughout the pandemic; which means that each airport loses - on average - half of its destinations. In absolute numbers, the average degree of airports worldwide is reduced from six to three. There are some important airports, which still have significantly higher degrees than the average; these airports will be analyzed individually below. The betweenness centrality of a node measures the fraction of shortest paths a node is located on. Intuitively, the larger the betweenness centrality, the more likely passengers will use an airport as a hub for transfer. It can be seen that the average betweenness centrality increases

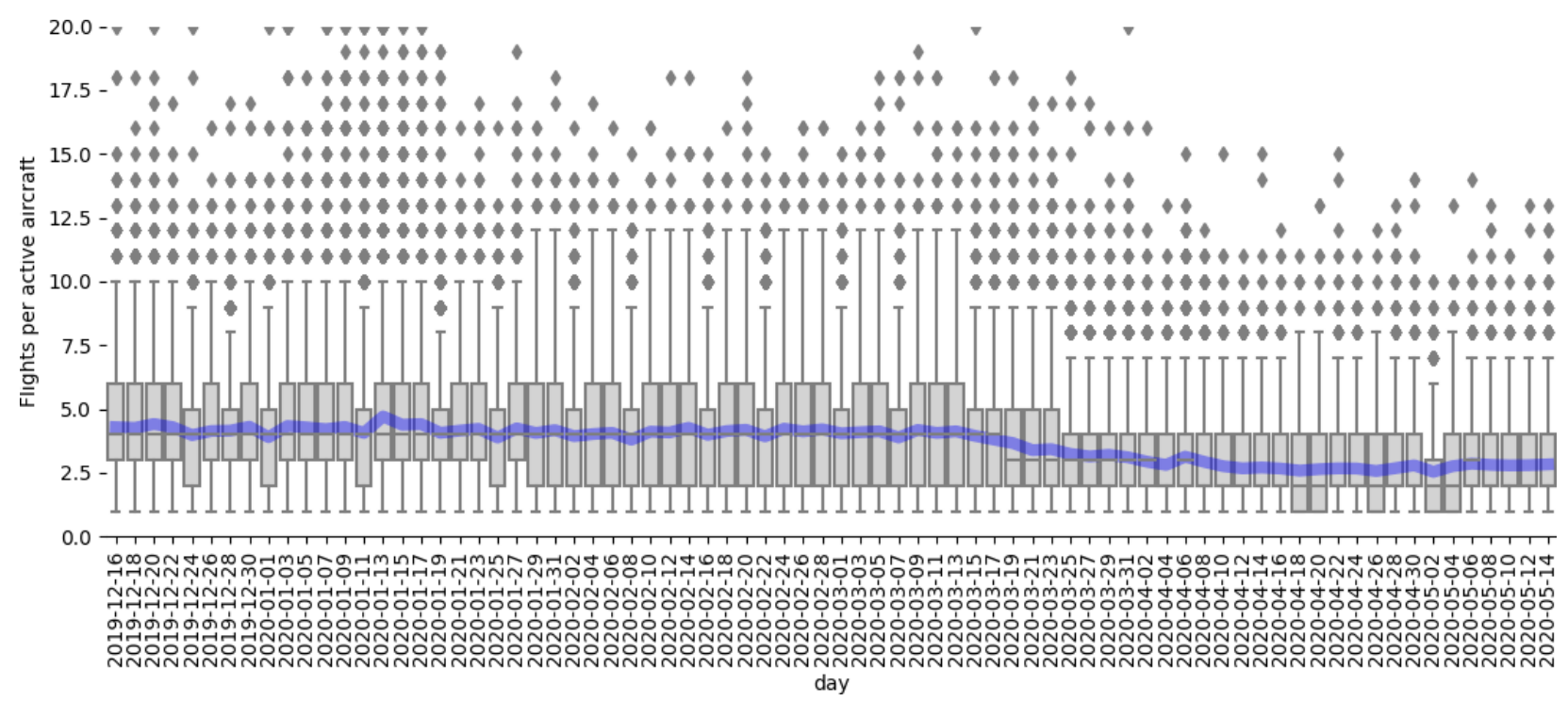

Figure 4. Evolution of the number of flights per aircraft per day. The boxes represent daily distributions of flights per aircraft (horizontal black line is the daily median). The shaded blue line is the average number of flights per aircraft per day.

Table 1. Overview on metrics in this study

\begin{tabular}{ll}
\hline \multicolumn{1}{c}{ Metric } & \multicolumn{1}{c}{ Description } \\
Degree & The number of direct neighbors of a node. \\
Betweenness & The frequency of which a node appears on all-pairs shortest paths. \\
Closeness & The sum of distances to all other nodes. \\
Clustering coefficient & How connected are the node's neighbors to each other? \\
Assortativity & The preference of a node to connected to other similar nodes. \\
Communities & Groups of nodes which are densely connected. \\
\hline
\end{tabular}



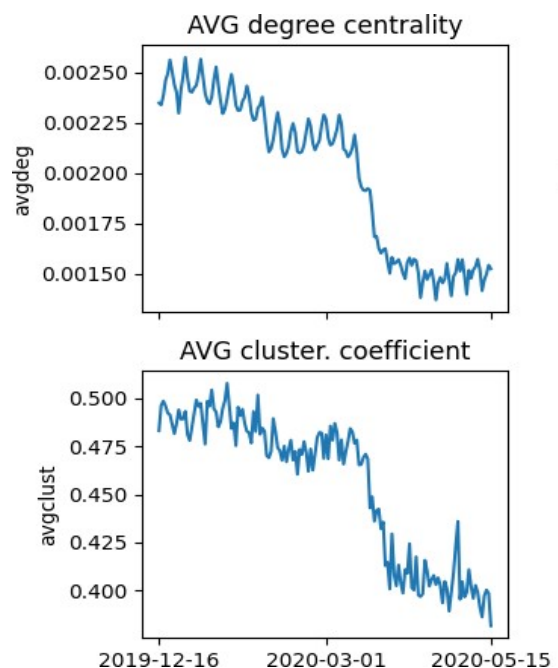

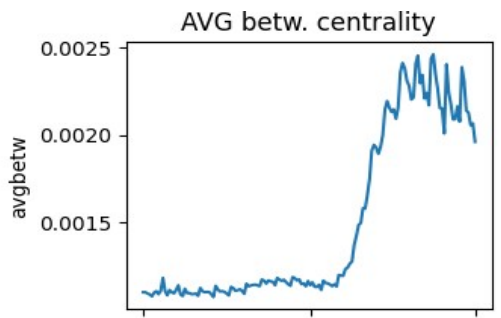

Assortativity

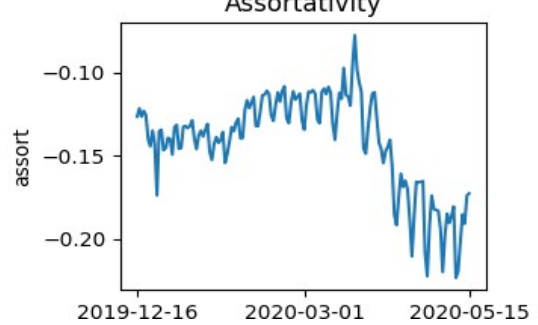

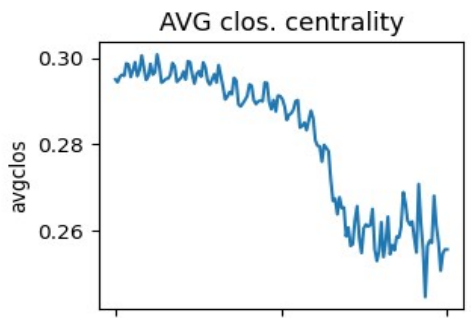

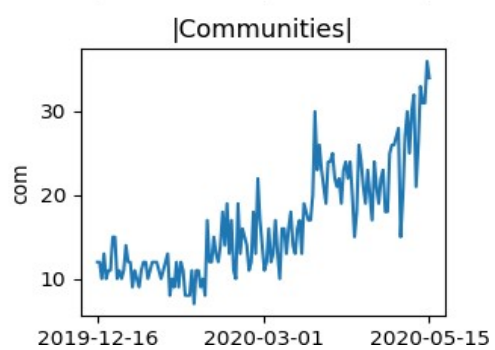

Figure 5. Evolution of selected airport network properties over time of the pandemic from December 2019 to May 2020.

significantly throughout the pandemic. The major reason is that a few of the biggest transfer hubs have been virtually shutdown, which increases the importance of other airports in the network; this effect will be analyzed further below as well. The closeness centrality of a node is computed as the reciprocal of the sum of the length of the shortest paths between the node and all other nodes in the network. Since smaller values of closeness centrality indicate further distance, as more links are removed (flight cancellations) from the network, efficient connectivity has gradually been falling apart. The average (global) clustering coefficient in a network is a proxy for transitivity in the network. Clustering is reduced, which is mainly caused by the decreased efficiency of the overall network. Assortativity, also referred to as assortative mixing, represents a node's preference to attach to others that are similar in some way. Here, we report the degree assortativity. A number of zero identifies non-assortative networks, while negative values imply that the network is completely disassortative. After a smaller peak in the transition around middle of May, the assortativity of the network is reduced, meaning that we have less hub-hub connections, but rather more hub-spoke connections in the network. Finally, the number of communities is increasing significantly throughout the pandemic, from 10 at the beginning to more than 30 in the middle of May.

While the previous discussion focuses on the network level, we report further results on the node-level perspective, i.e., with a focus on individual airports. We selected the network metrics degree and betweenness, given their importance for complex systems of all types. Figure 6 visualizes the temporal evolution of degree and betweenness for selected airports (color) and all
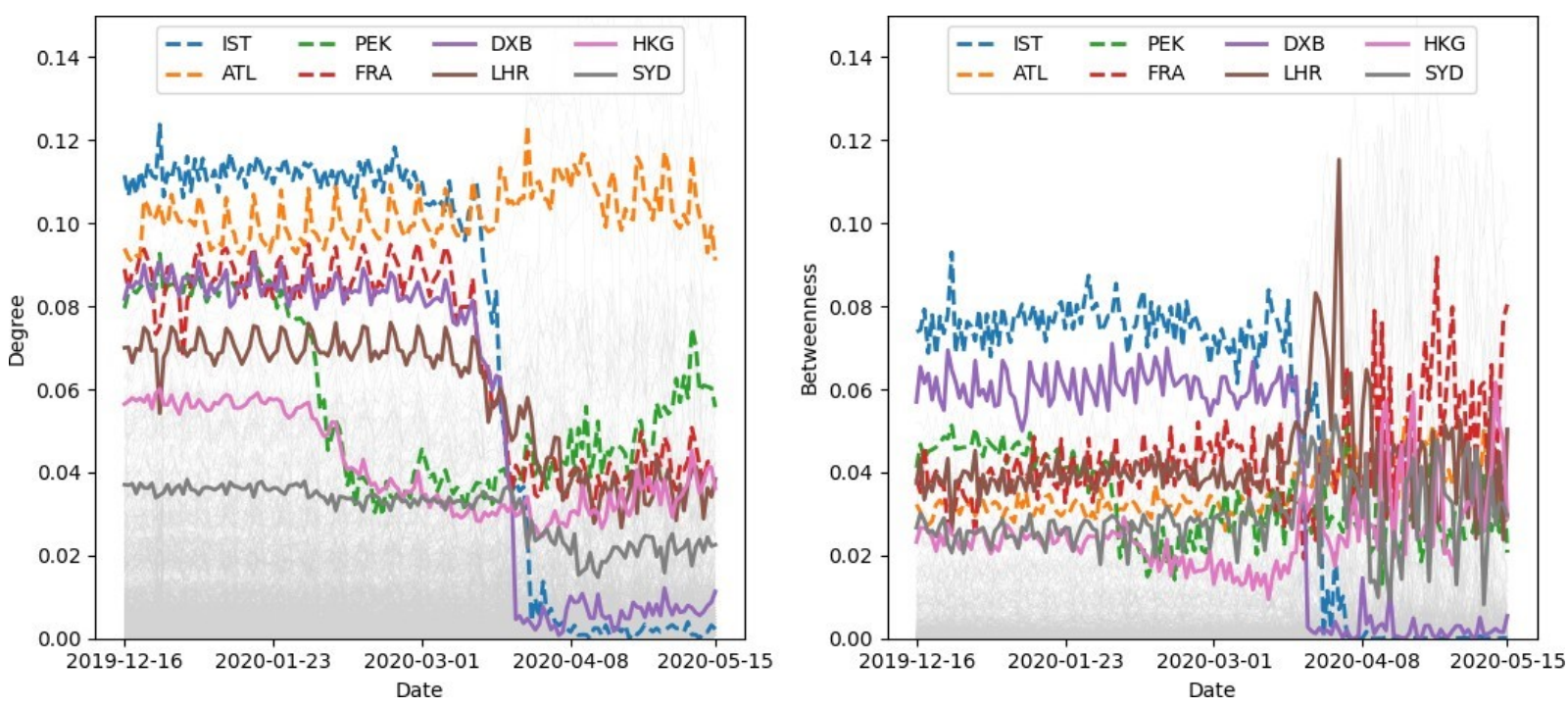

Figure 6. Evolution of node degree and node betweenness for selected airports (colored) and all other airports in the network (grey). 
other airports (grey). We selected eight airports by taking into account the total number of passengers in 2019 and considerations of spatial diversity. Regarding the degree centrality, we find that most of the larger airports face a significant drop in the number of destinations. This particularly holds true for the two major hub airports Istanbul Airport (IST) and Dubai International Airport (DXB) that both virtually dropped to a status of inoperativity. Being highly important for connecting Europe and Asia/Oceania, the discontinuation of these two airports makes sense, intuitively. Nevertheless, we can see that other airports maintain a high number of destinations. For instance, Hartsfield-Jackson Atlanta International Airport (ATL), which is a central hub for domestic transportation in the United States has been relatively unaffected by the pandemic so far. Finally, we can see that some airports are on the way to regain their original degree; for instance, Beijing Capital International Airport (PEK) has recovered about half of its originally-lost destinations (yet, at a much lower frequency, with limits on the number of international flights per destination and week). Regarding the betweenness centrality, two phases can be distinguished. Before the pandemic, the betweenness centrality of airports was rather stable, with IST and Dubai International Airport (DXB) being the major components keeping the (inter-continental) system together. After the changes to the airport network, induced by countries' travel bans, the system has been in a highly fragile state, measured by the variance in the betweenness of nodes. For the whole period of April and May, there has been no single (long-term) outstanding node with high betweenness; in the contrary, the betweenness of nodes varies tremendously even within one week by up to one order of magnitude. Such changes in the importance can only be observed in systems with a high degree of chaos. Moreover, this fluctuation in node importance suggests that it is arduous for passengers right now to make informed choices on their travels, especially when involving more than one hop.

In order to better understand the changes in node importance, we investigate the top-ranked betweenness nodes further. Figure 7 shows the nodes that have been ranked Top 30 in the first week of our study period and/or in the last week of our study period. Those nodes that were in the Top 30 in both weeks are visualized in black color. A few airports on each continent can be found to be rather stable regarding the ranking (yet, their actual betweenness values still vary significantly). Airports which have disappeared from the Top 30 are highlighted in red and airports which have newly appeared in the Top 30 are shown in blue color. One interesting case is that of Hamad International Airport (DOH), which remained highly central, while DXB was completely taken out. This shows that competition still plays an important, yet counter- productive role in the pandemic. If one wanted to cut down international connections successfully, then both (strongly competing) hubs should have reduced their criticality in the network (Sun et al. 2017a). The way it is right now, it seems like DOH might just use the pandemic to further

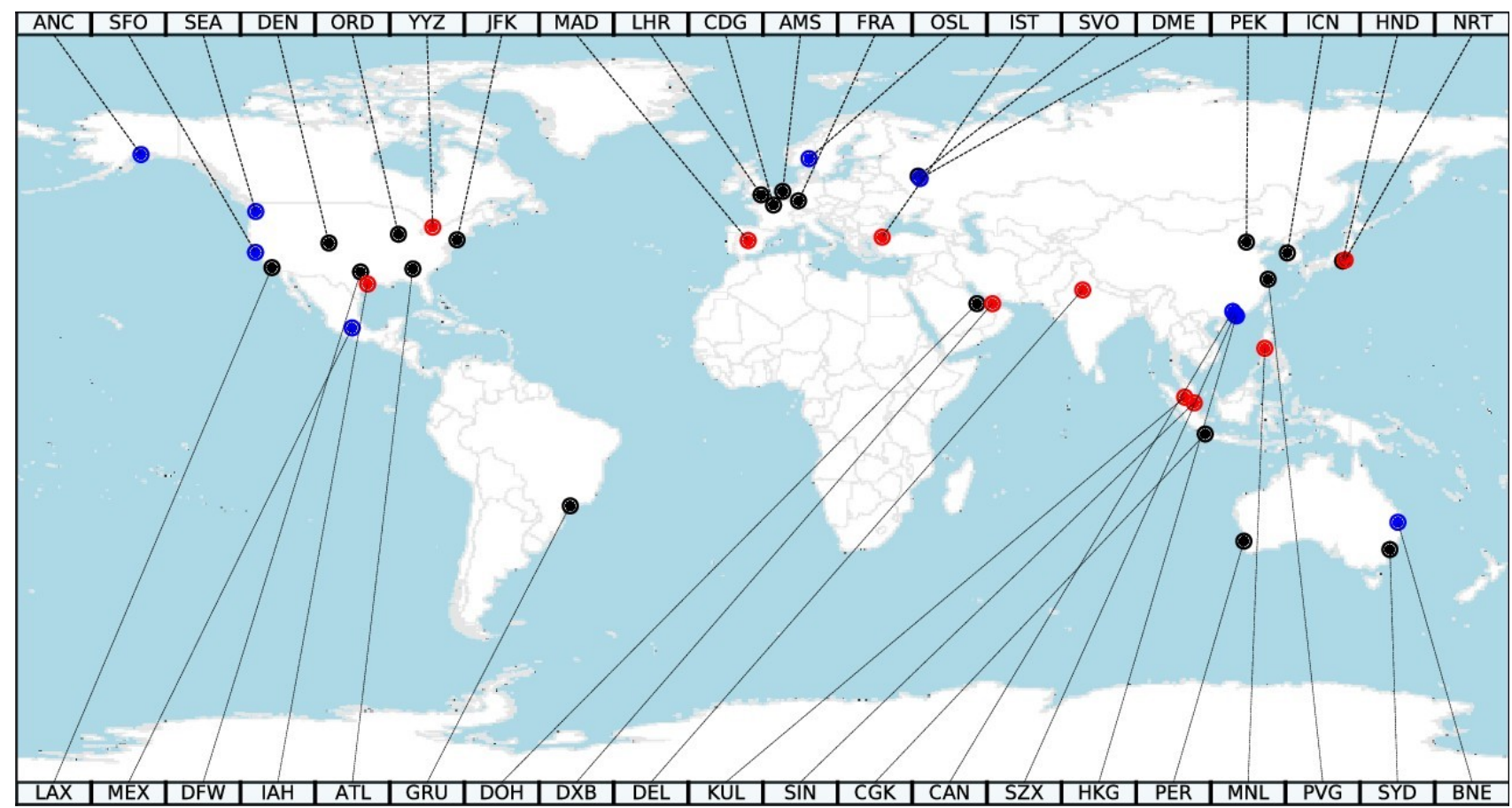

Figure 7. Visualization of nodes with high betweenness and how they evolved during the pandemic. Airports in black color are those in the Top 30 for both periods (before and during the pandemic). Airports in red color are those with vanishing betweenness, i.e., they were in the Top 30 before the pandemic, but not anymore. Airports which newly entered the Top 30 are visualized in blue color. 
consolidate its strength and market position as an intercontinental hub.

\subsection{International country network}

The experiments so far have analyzed the global air transportation system at airport level. Node aggregations often lead to additional insights regarding the system. Since the pandemic shows stronger impacts on international passenger traffic than domestic passenger traffic, we discuss the so-called country network (Wandelt and Sun 2015) below, where nodes are countries and links represent the direct flights between countries. The country network is created from the airport network by merging all airports that are located in the same country. Self-loops (induced by domestic connections) are removed. The number of flights between a pair of countries is computed as the sum of flights over all airport pairs for these two countries.

Figure 8 depicts the international country network on four selected dates before and throughout COVID-19. In total, we have data for 213 countries within our study. These snapshots reveal the connectivity patterns of individual countries and how they changed over time. Each country is represented by a node whose location in set to the median location of all airports in the country. We can see that some countries are still highly connected with other countries, e.g., the United States, Canada, and Mexico. Other countries have significantly reduced their connectivity, e.g., countries in Europe. As previously noted for the airport network, it is apparent that the countries in South America and Africa have dramatically reduced their connectivity to selected destination countries, compared to countries in North America.

In our next analysis, the international country network is investigated from a complex system perspective. We have computed the measure of six network properties; the results are shown in Figure 9. It can be seen that most of the trends coincide with the airport network's statistics. Most transitions between before travel bans and after travel bans are more sudden, with less variation, compared to the airport network. One interesting difference is in the measure of assortativity. For the airport network, the assortativity was decreasing, which means that high-degree airports are increasingly connected to low-degree airports during the pandemic. For the country network, on the other hand, there is a trend that high-degree countries are more actively connected to other high-degree countries. This shows that there is a preference between countries to connect to other countries with more connections during the pandemic. There is, nevertheless, a strong variance in this pattern. Finally, the number of communities is increasing as well, which means that not only airport groups from communities have preferential connections, but so do countries.
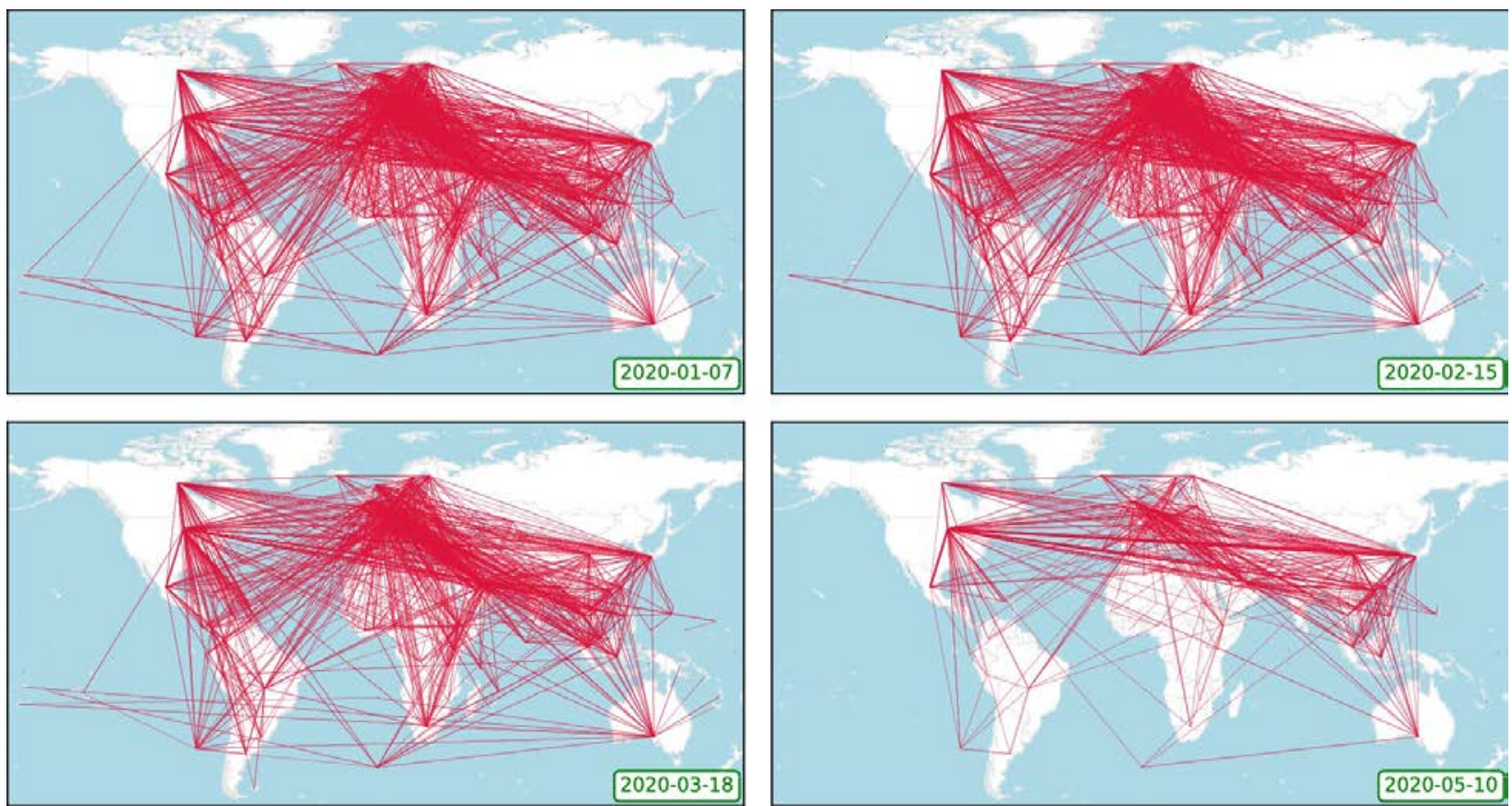

Figure 8. International country network on four select dates before and during the COVID-19 pandemic. Nodes represent countries and links represent direct flight connections between countries; the width of a link corresponds to the number of direct flights between the countries. Self-loops (i.e., domestic flights) are not shown. 

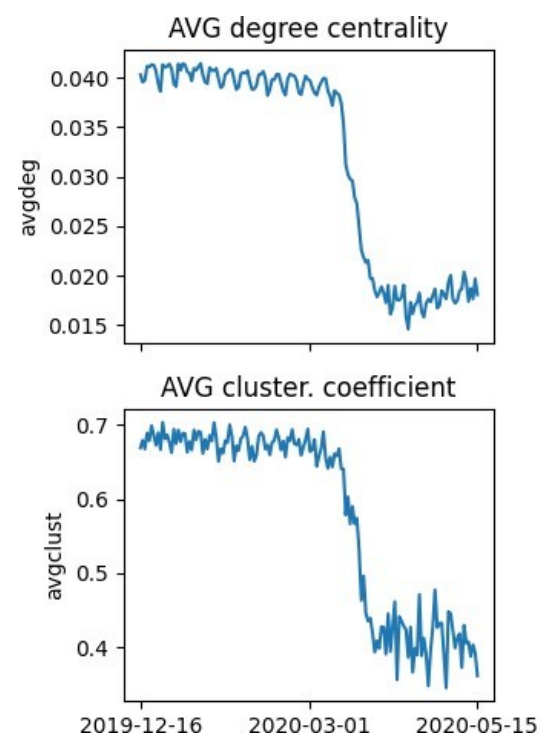
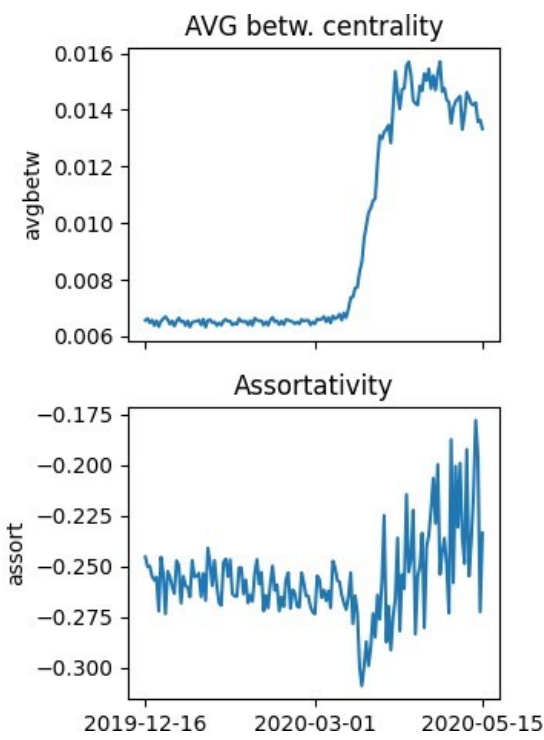
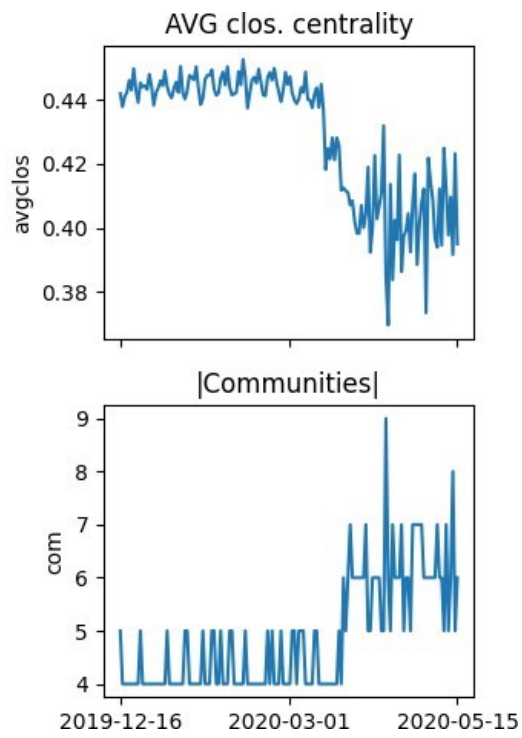

Figure 9. Evolution of selected country network properties over time of the COVID-19 pandemic from January 2020 to May 2020.
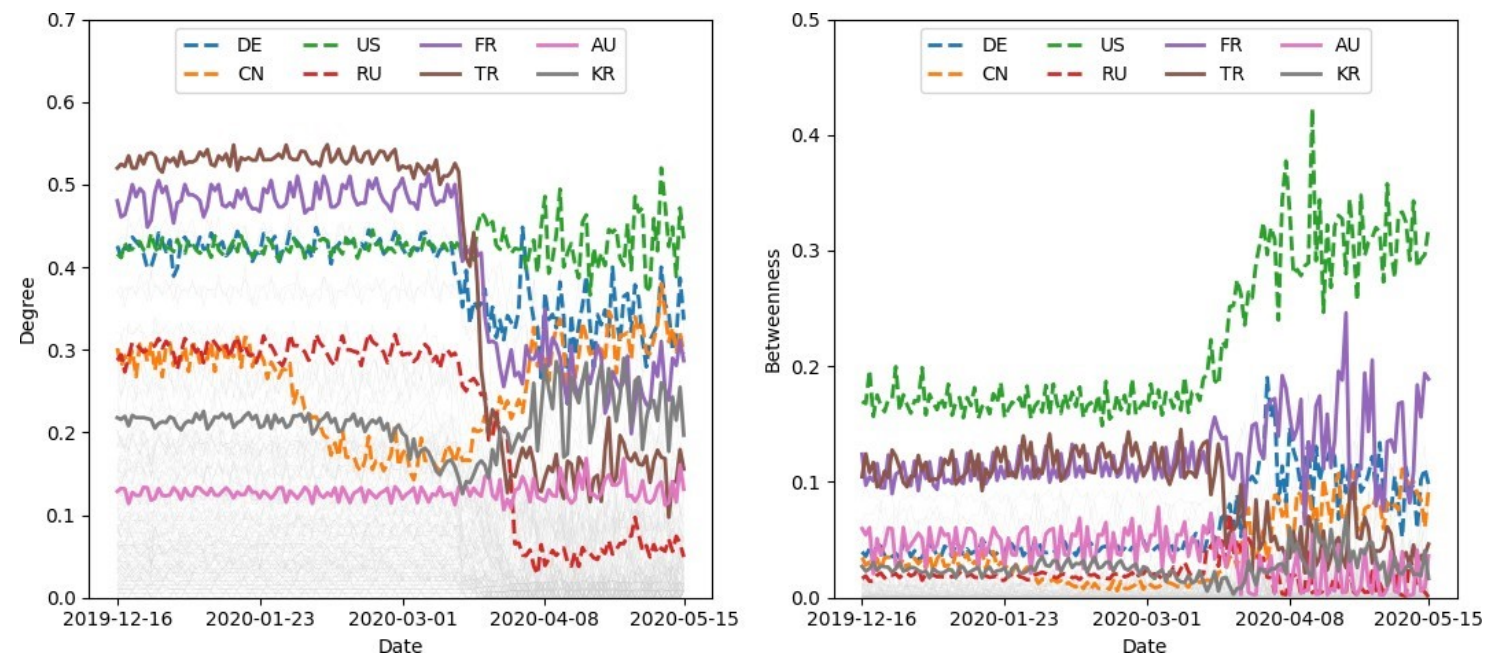

Figure 10. Evolution of the degree centrality and betweenness centrality for selected countries (colored) and all other countries in the network (grey) in the international country network during the COVID-19 pandemic from December 2019 to May 2020.

Next, we shift the discussion from a network level to a node-level perspective, i.e., with a focus on individual countries. Figure 10 reports the temporal evolution of two network metrics, degree and betweenness. Regarding the degree centrality, the changes in connectivity are not as extreme as in the airport network. Turkey (TR), Russia (RU) and France (FR) have experienced a significant reduction in destination countries; the other four selected countries have not been severely affected by the pandemic so far. Regarding the betweenness centrality, an interesting observation can be made for the United Stated (US). Despite widely-communicated travel bans, the country increased its importance in the country network throughout the pandemic. The high betweenness value of up to 0.4 is astonishing; this indicates that the US would be on up to $40 \%$ of all shortest paths through the country network. Therefore, from the complex-network perspective, the United States (US) has gone through the pandemic rather well so far. It is difficult to state a single reason for this observation; it might be related to the large number of US nationals spread around the world, who are still allowed to come back, despite active travel bans for larger parts of the external population. Either way, this phenomenon should be analyzed and explained further in future research.

\subsection{Domestic airport networks}

The international role of countries in the country network was one perspective of the air transportation system. Another 
perspective, which is taken in this section, is the role of air transportation in domestic transportation systems, i.e., within specific countries/regions. In the following, we discuss the domestic airport network, where nodes are airports within a specific country/region and links represent the direct flights between these airports. Analyzing these networks is of particular importance, given that the travel bans are mainly implemented at inter-country level. Therefore, it is interesting how air transportation has been affected by the pandemic within countries.

Figure 11 shows the snapshots of four selected domestic networks before the pandemic and throughout the pandemic. We have selected Australia, China, Europe and United States for comparison; the region Europe was selected instead of an individual country, since the area of Europe is of the same magnitude as the other countries. Australia has experienced a significant breakdown of its domestic airport network; in the latest network, only few coastal regions are still connected by air transportation, with almost no cross-country flights remaining. China had a visible, but not extensive reduction of air traffic in February, during the major part of its domestic lock-down. Since then, the airport network has largely recovered to its original connectivity. Europe has probably undergone the most significant domestic changes of all four, with the latest network snapshot resembling a very sparse connectivity compared to the situation before the pandemic. Finally, the United States has also undergone changes in the latest snapshot, but significantly less severe than Europe. These snapshots reveal that the air transport lock-down in domestic networks happens to a different degree and is partially un-synchronized, with China having a head start (almost recovered by now) and the United States locking-down relatively later. However, such analysis should take into account
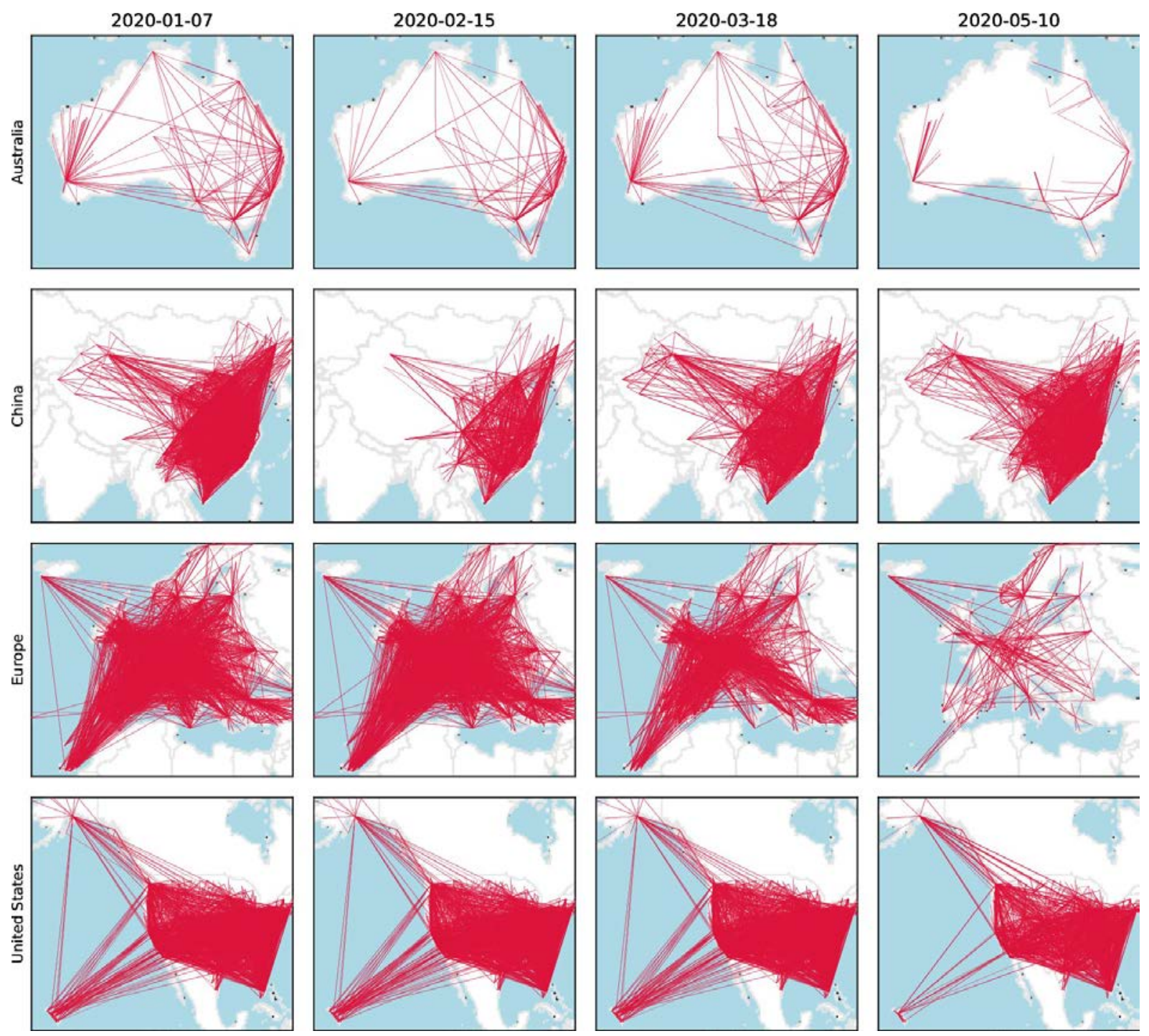

Figure 11. Evolution of four domestic airport networks (China, Europe, United States, and Australia) during the COVID-19 pandemic from January 2020 to May 2020. 
that the ground transportation systems in all four regions are significantly different. For instance, Europe and China have a well-established rail / high-speed rail system, which could possibly compensate for locking down air transportation. The United States and Australia, on the other hand, have to rely on air transportation for long-distance travel.

\section{Discussion and conclusions}

In a final experiment, we aim to understand the relations between the reduction of the number of flights and the number of confirmed cases in a country. Essentially, the idea of cutting of flights is to reduce/stop the spread, be it from external regions (international flights) or within a country (domestic). Figure 12 shows the temporal evolution of domestic and international flights (red) versus the number of cases in selected countries. In total, we report these results for twelve countries; these countries were ranked and selected based on the total number of infections on May 24th, 2020. The charts reveal an interesting observation between the effective reduction in flights and the beginning of the surging number of reported cases. Particularly, for almost all cases, the flight reductions largely coincide with the time the first cases are reported in the country. Given an incubation period of fourteen or even more days, it can be concluded that most countries in fact reduced their flight operations just too late: the virus had already been imported into the country when the restrictions took full effect. Moreover, in most cases the reduction of domestic flights followed the reduction of international flights with a significant delay. Without that delay, the spread of the disease inside a country might have been reduced earlier. The current study provides a comprehensive empirical analysis on the impact of the COVID-19 pandemic on air transportation systems, based on a multi-granularity network analysis, covering worldwide airport networks, international country networks, and domestic airport networks. The time scale ranges from January 2020 to May 2020 of the COVID-19 pandemic outbreak. We summarize the major findings of our study below:
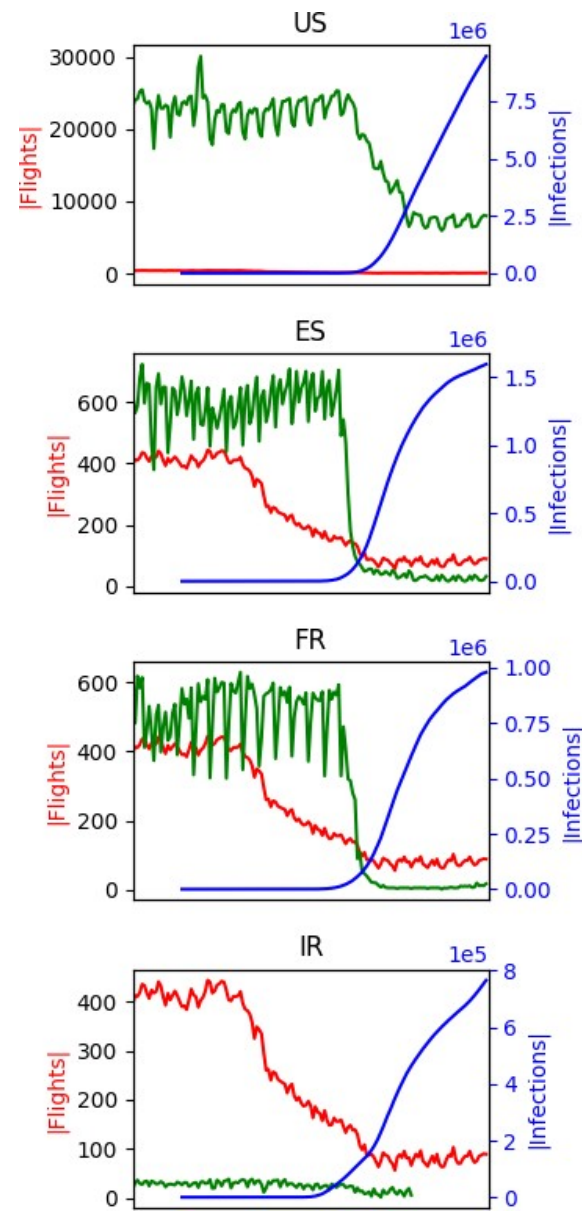
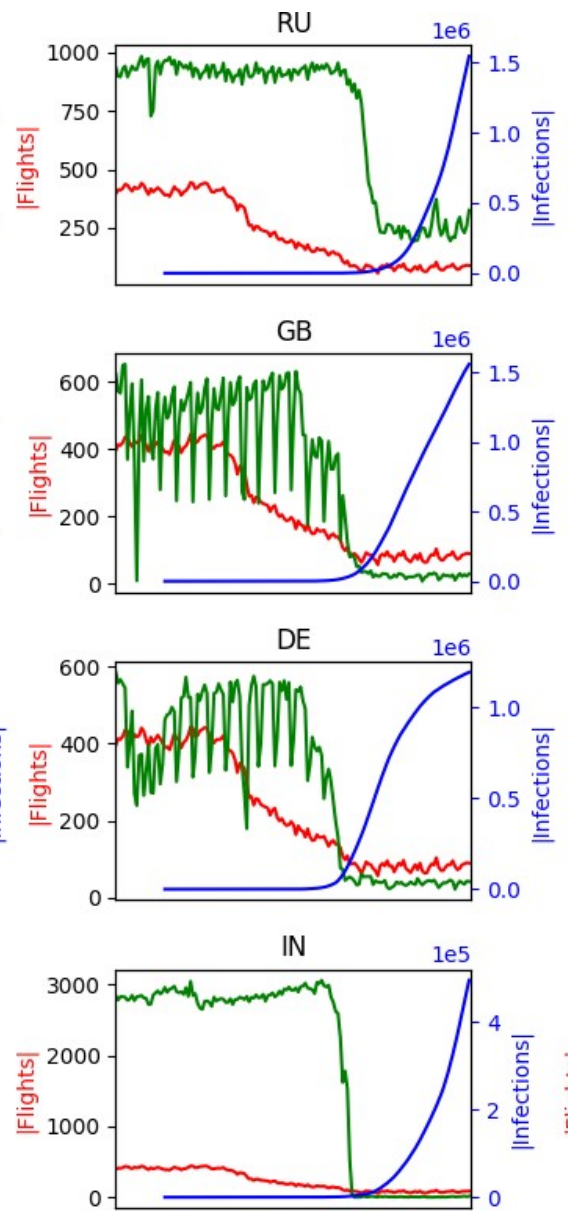
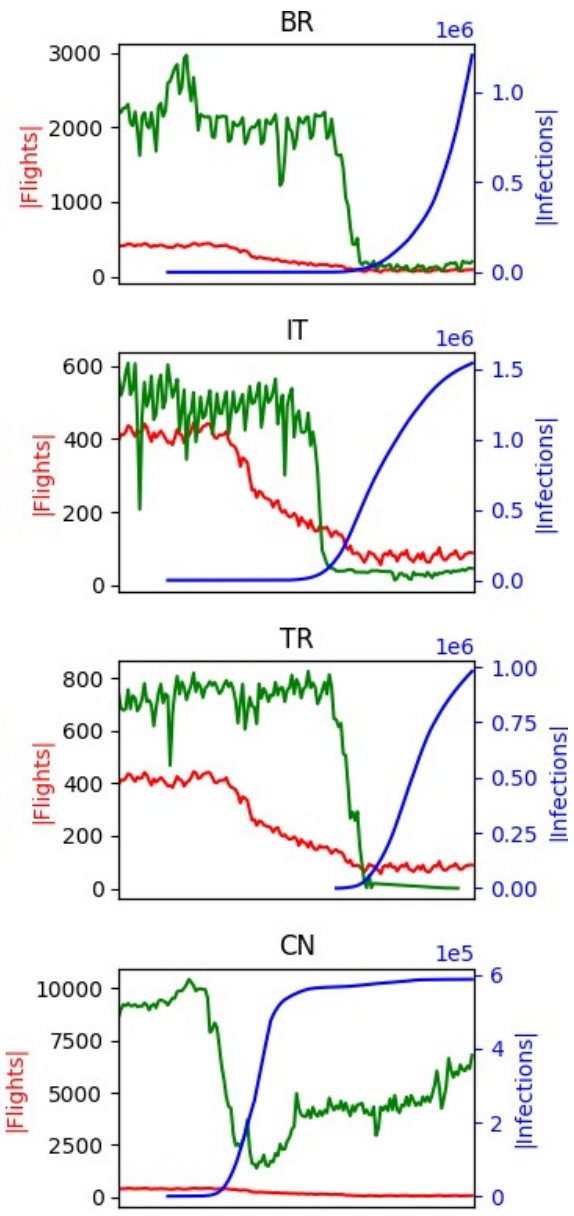

Figure 12. Evolution of domestic and international flights versus the number of infected cases in selected countries. Domestic flights are shown as green lines and international flights as a red line; The reported number of infected cases in a country is shown as a blue line. The $x$-axis is time. 
1. We observed from the worldwide airport network that the Southern hemisphere has been more affected than the Northern part. More strikingly, the worldwide airport network seemed to have been largely unchanged for the first 2-3 months of the COVID-19 pandemic. The evolution of the number of OD pairs and the number of aircraft in operation showed that the aviation industry has reacted to the pandemic with a delay of about two months, and the opportunity to avoid the COVID19 from a local burst to a global pandemic was missed due to the postponement. Given existing research (Brockmann and Helbing 2013) and the degree of knowledge about the extent of the disease in January 2020, one would have expected a much more coordinated and informed global response.

2. The slight reduction in the distance between OD pairs showed that flight restrictions were mainly imposed on long-distance international flights; accordingly, the impacts of the COVID- 19 pandemic on international flights have been much stronger than on domestic flights. While this seems to be a natural move, the role of aviation for spreading the virus in domestic networks is probably underestimated. Taking the United States as an example, there exists no real alternatives to air transport when it comes to traveling from the East-coast to the West-coast. Accordingly, if the domestic airport network of the United States is still largely active, one needs to be aware that domestic flights are not immune to disease propagation. Essentially, our analysis reveals a largely-uncoordinated response to COVID-19.

3. Six commonly-used complex network metrics in the worldwide airport network showed that each airport lost $50 \%$ of its connections on average, efficient network connectivity was further reduced, and the world increasingly created virtual borders between groups of airports throughout the pandemic. In particular, two major hubs connecting Europe and Asia/Oceania (IST and DXB) essentially dropped to the status of inoperativity; however, a central hub for the United States (ATL) has been largely unaffected throughout the pandemic. The fluctuation in node importance suggests that competition may still play an important, yet counter-productive role in the pandemic spreading.

4. Analysis of country networks focusing on the international passenger traffic of 213 countries worldwide showed that connectivity patterns for individual countries are heterogeneous and fluctuate depending the COVID-19 situation. While most of the evolution trends coincide with the worldwide airport network statistics, the difference in the assortativity metric showed that high-degree countries are more actively connected to high-degree countries, sharing a similar preferential attachment mechanisms during the pandemic for the international country network; on the contrary high-degree airports are increasingly connected to low-degree airports in the worldwide airport network.

5. Nodal-level analysis of the international country network revealed that the importance of countries is changing significantly during the pandemic. Notably, the United States became one of the most critical countries in May 2020, if measured according to standard complex network indicators. These findings are somewhat contrary to the general belief that the United States administration locked all borders. Most of the other countries in the world experienced drastic reductions in their connectivity, due to the severe consequences of the pandemic.

6. The evolution of domestic airport networks focusing on the domestic passenger traffic for representative countries/regions showed different degrees of lock-down and quite obvious patterns of asynchronization: Europe has undergone probably the most significant changes regarding network connectivity, while the United States suffered less severely and China seems to be to have a head start towards recovery. Accordingly, the evolutionary dynamics of domestic airport networks are tightly correlated with the COVID-19 situation in specific countries.

Our current study suffers a few limitations, partially induced by lack of data. We use flight schedule data in the current study, we do not have access to real passenger data in the current stage. The common way of average load factor approximation is highly inaccurate because of various repatriation flights, medical support flights, charted flights etc. Moreover, we did not distinguish passenger flights and cargo flights, since it is not clear how to distinguish between aircraft transporting passengers from those transporting cargo. Furthermore, several airlines are using passenger aircraft to transport cargo during the COVID19 pandemic. And one has to keep in mind that the COVID-19 pandemic is not over yet; recovery not included plus future, multiple waves. Future work should look at airline-specific analysis, i.e., how airlines react to the COVID-19 pandemic in order to minimize their losses. Finally, future work should investigate possible impacts and scenarios of COVID-19 on air transportation. For instance, it would be of great interest to the community to foresee whether the pandemic will induce a rather V-shaped or U-shaped return to normality; where the latter would show a much longer period of reductions. Similarly, it will be interesting to see how different parts of the world will be connected in the future, with pairwise travel restrictions possibly leading to so-called travel bubbles. 


\section{Acknowledgements}

This study is supported by the National Natural Science Foundation of China (Grant No. 61861136005, No. 61650110516, and No. 71731001).

\section{References}

Bogoch, I. I., Creatore, M. I., Cetron, M. S., Brownstein, J. S., Pesik, N., Miniota, J., Tam, T., Hu, W., Nicolucci, A., Ahmed, S., Yoon, J. W., Berry, I., Hay, S. I., Anema, A., Tatem, A. J., MacFadden, D., German, M., Khan, K., 2015. Assessment of the potential for international dissemination of Ebola virus via commercial air travel during the 2014 west African outbreak. The Lancet 385, 29-35.

Brockmann, D., Helbing, D., 2013. The hidden geometry of complex, network-driven contagion phenomena. Science 342, 1337-1342.

Christidis, P., Christodoulou, A., 2020. The predictive capacity of air travel patterns during the global spread of the COVID-19 pandemic: Risk, uncertainty and randomness. International Journal of Environmental Research and Public Health 17, 3356.

Colizza, V., Barrat, A., Barthelemy, M., Vespignani, A., 2006. The role of the airline transportation network in the prediction and predictability of global epidemics. Proceedings of the National Academy of Sciences 103, 2015-2020.

Diaconu, L., 2012. The evolution of the European low-cost airlines business models. Ryanair case study. Procedia-Social and Behavioral Sciences 62, 342-346.

Gilbert, M., Pullano, G., Pinotti, F., Valdano, E., Poletto, C., Boëlle, P. Y., D'Ortenzio, E., Yazdanpanah, Y., Paul Eholie, S., Altmann, M., Gutierrez, B., Kraemer, M. U. G., Colizza, V., 2020. Preparedness and vulnerability of African countries against importations of COVID-19: A modelling study. The Lancet 395, 871-877.

Iannelli, F., Koher, A., Brockmann, D., Hövel, P., Sokolov, I. M., 2017. Effective distances for epidemics spreading on complex networks. Physical Review E 95, 012313.

Janic, M., 2000. An assessment of risk and safety in civil aviation. Journal of Air Transport Management 6, 43-50.

Lee, D. S., Fahey, D. W., Forster, P. M., Newton, P. J., Wit, R. C., Lim, L. L., Owen, B., Sausen, R., 2009. Aviation and global climate change in the 21st century. Atmospheric Environment 43, 3520-3537.

Likhacheva, A., 2006. SARS Revisited. American Medical Association Journal of Ethics 8, 219-222.

Sun, X., Wandelt, S., Cao, X., 2017a. On node criticality in air transportation networks. Networks and Spatial Economics 17, 737-761.

Sun X., Wandelt S., Zanin M., 2017b. Worldwide air transportation networks: A matter of scale and fractality? Transportmetrica A-Transport Science 13, 607-630.

Wandelt, S., Sun, X., 2015. Evolution of the international air transportation country network from 2002 to 2013. Transportation Research Part E: Logistics and Transportation Review 82, 55-78.

Wandelt S., Sun X., Feng D., Zanin M., Havlin S., 2018. A comparative analysis of approaches to network-dismantling. Scientific Reports 8, 13513.

Wandelt, S., Sun, X., Zhang, J., 2019. Evolution of domestic airport networks: A review and comparative analysis. Transportmetrica B: Transport Dynamics 7, 1-17.

Zaki, A. M., van Boheemen, S., Bestebroer, T. M., Osterhaus, A. D., Fouchier, R. A., 2012. Isolation of a novel coronavirus from a man with pneumonia in Saudi Arabia. New England Journal of Medicine 367, 1814-1820.

Zhang, Y., Zhang, A., Wang, J., 2020. Exploring the roles of high-speed train, air and coach services in the spread of COVID19 in China. Transport Policy 94, 34-42. 